\title{
Errata
}

\section{Erratum. Celastrol-Induced Weight Loss Is Driven by Hypophagia and Independent From UCP1. Diabetes 2018;67:2456-2465}

Katrin Pfuhlmann, Sonja C. Schriever, Peter Baumann, Dhiraj G. Kabra, Luke Harrison, Sithandiwe E. Mazibuko-Mbeje, Raian E. Contreras, Eleni Kyriakou, Stephanie E. Simonds, Tony Tiganis, Michael A. Cowley, Stephen C. Woods, Martin Jastroch, Christoffer Clemmensen, Meri De Angelis, Karl-Werner Schramm, Michael Sattler, Ana C. Messias, Matthias H. Tschöp, and Paul T. Pfluger

https://doi.org/10.2337/db19-er03a

In the article listed above, ERX was erroneously omitted from the duality of interest for Matthias H. Tschöp. The statement should have read "M.T.H. is a scientific advisor to Novo Nordisk and ERX." The authors apologize for the error.

The online version of the article (https://doi.org/10.2337/db18-0146) has been updated to correct this omission.

\section{Erratum. Glucolipotoxicity-Inhibited miR-299-5p Regulates Pancreatic $\beta$-Cell Function and Survival. Diabetes 2018;67:2280-2292}

Qiqing Huang, Weiyan You, Yating Li, Yi Sun, Yuncai Zhou, Yan Zhang, Dechen Liu, Shanshan Zhan, Yunxia Zhu, and Xiao Han https://doi.org/10.2337/db19-er03b

In the article listed above, the funding grants were erroneously given in the wrong order as "This study was supported by research grants from the National Natural Science Foundation of China (81670703 to Y. Zhu and 81420108007 to X.H.) and the National Key Research and Development Program of China (2016YFC1304804) to X.H." The correct order is "This study was supported by research grants from the National Key Research and Development Program of China (2016YFC1304804) to X.H. and the National Natural Science Foundation of China (81420108007 to X.H. and 81670703 to Y. Zhu)." The editors apologize for this error.

The online version of the article (https://doi.org/10.2337/db18-0223) has been updated to correct this error. 